\title{
INFLOW AND OUTFLOW PROBLEMS IN THE HALF SPACE FOR A ONE-DIMENSIONAL ISENTROPIC MODEL SYSTEM OF COMPRESSIBLE VISCOUS GAS
}

\author{
AKITAKA MATSUMURA*
}

\begin{abstract}
We consider asymptotic behaviors in time of solutions to the initial boundary value problems in the half space for a one-dimensional isentropic model system of compressible viscous gas. In particular, we focus our attention on inflow(or outflow) problems where the velocity on the boundary is given as a constant inward(or outward) flow, and try to classify all asymptotic behaviors of the solutions. It turns out that depending on the data both on the boundary and at far field (especially depending on whether the state is subsonic, transonic, or supersonic), the asymptotic state variously consists of rarefaction waves, viscous shock waves, and also stationary boundary layer. Moreover, we give a survey of our recent results on some particular cases which justify our classification.
\end{abstract}

1. Introduction. The one-dimensional and isentropic motion of compressible viscous gas which fills the half space is described by the following system in the Eulerian coordinates:

$$
\left\{\begin{array}{l}
\rho_{t}+(\rho u)_{x}=0 \\
(\rho u)_{t}+\left(\rho u^{2}+p(\rho)\right)_{x}=\mu u_{x x}, \quad x>0, t>0 \\
p(\rho)=a \rho^{\gamma}
\end{array}\right.
$$

where $\rho(>0)$ is the density, $u$ is the velocity, $p$ is the pressure, all coefficients $\mu(>0)$, $a(>0)$, and $\gamma(\geq 1)$ are assumed to be constant. We study the initial boundary value problems to the system (1.1) with the initial data

$$
(\rho, u)(0, x)=\left(\rho_{0}, u_{0}\right)(x), \quad x>0, \quad \inf _{x>0} \rho_{0}(x)>0,
$$

the boundary condition at far field $x=+\infty$

$$
\lim _{x \rightarrow \infty}(\rho, u)(t, x)=\left(\rho_{+}, u_{+}\right), \quad t>0,
$$

and also one of the following three types of conditions on the boundary $x=0$.

Case 1 (zero velocity on the boundary):

$$
u(t, 0)=0, \quad t>0 .
$$

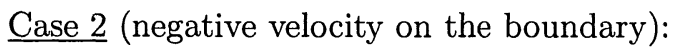

$$
u(t, 0)=u_{-}<0, \quad t>0
$$

$\underline{\text { Case } 3}$ (positive velocity on the boundary):

$$
\left\{\begin{array}{l}
u(t, 0)=u_{-}>0, \\
\rho(t, 0)=\rho_{-}>0,
\end{array} \quad t>0 .\right.
$$

*Department of Mathematics, Graduate School of Science, Osaka University, Osaka 560-0045, Japan. 
Here $\rho_{ \pm}$and $u_{ \pm}$are prescribed constants, and we of course assume the initial data satisfy the boundary conditions (1.3) and one of (1.4) as compatibility condition. It is noted that the Case 1 means the wall is impermeable, and the Case 2 (resp. Case 3) means the outflow (resp. inflow) constantly exists on the wall. It is also noted that in the Cases 1 and 2 the condition on the density can't be imposed, but in the Case 3 it has to be imposed, so that the corresponding problem to the first equation of (1.1) (conservation of mass density) is well posed as a hyperbolic equation for the mass density $\rho$. In what follows, we call the initial boundary value problem $(1.1) \sim(1.4)_{1}$, $(1.1) \sim(1.4)_{2}$, and $(1.1) \sim(1.4)_{3}$, the impermeable wall problem, outflow problem, and inflow problem, respectively. In this article, we are interested in the time-global existence of the solutions of these three types of initial boundary value problems, especially the large time asymptotic behaviors of the solutions in connection with the various combinations of the data on the boundary and at far field.

There have been many works on the asymptotic behaviors of solutions to the Cauchy problems for the system (1.1) where the boundary conditions at far fields $x= \pm \infty$ are given by

$$
\lim _{x \rightarrow \pm \infty}(\rho, u)(t, x)=\left(\rho_{ \pm}, u_{ \pm}\right), \quad t>0 .
$$

Refer to [9] [11], [14], [7], etc., and the references therein. All these results show that the large time asymptotic behaviors of solutions of the Cauchy problem with (1.5) are basically same as that of Riemann problem to the hyperbolic part of (1.1)(Euler equation), if only we replace the shock wave with discontinuity by the corresponding smooth viscous shock wave. Hence the asymptotic behaviors are naturally classified into eight different patterns of combination of the rarefaction and viscous shock waves. On the other hand, in the cases of IBVP, the influence of viscosity is expected to emerge not only in smoothing effect on discontinuous shock wave, but also in forming a boundary layer. As for the question when the boundary layer forms, we propose a criterion as follows by considering the Riemann problem for the Euler equation, where the initial right state $\left(\rho_{+}, u_{+}\right)$is given by the far field state (1.3), and the left state $\left(\rho_{-}, u_{-}\right)$is given by all possible state which is consistent with the boundary condition (1.4) on $x=0$ (that is, in the cases of the impermeable wall and outflow problems, $\rho_{-}$is freely given). When the left state is uniquely determined so that the value on the boundary $x=0$ of the solution of the Riemann problem is consistent with the boundary condition, that is when the solution consists of only elementary waves with positive speed, we expect no boundary layers emerge. On the other hand, when the value of the Riemann problem's solution on the boundary is not consistent with the boundary condition for any admissible left state, that is the case when the solution includes an elementary wave with negative speed or a stationary shock wave, we expect a boundary layer which smoothly but steeply compensate the gap comes up. Roughly speaking, inconsistency of the incoming or standing hyperbolic waves with the boundary data as the hyperbolic-parabolic system does form a boundary layer. Thus, we can easily imagine the situation crucially depends on whether the state either on the boundary and at far field is subsonic, transonic, or supersonic, because the characteristic wave speeds of the Euler equation are given by $u \pm c(\rho)$, where $c(\rho)$ is so called the sound speed. Recently the impermeable wall problems are investigated by Matsumura \& Mei [8] and Matsumura \& Nishihara [12], and it turns out that the asymptotic value of $\rho_{-}$is uniquely determined by the given far field data $\left(\rho_{+}, u_{+}\right)$so that no boundary layers appear and the solution eventually tends toward an outgoing rarefaction wave in the case $u_{-}<u_{+}([12])$, and a properly shifted viscous shock 
wave in the case $u_{-}>u_{+}([8])$. This means the asymptotic behaviors of solutions are basically classified only into two patterns, making a remarkable contrast to the Cauchy problem. On the other hand, the inflow and outflow problems are expected to be far more complicated, and in fact, there have been few results on them. In this paper, we construct the boundary layer by the stationary solution, and try to classify the all possible large-time asymptotic behaviors of solutions of the inflow and outflow problems through the criterion mentioned above. Moreover, we give a survey of our just recent results on some typical cases, which justify our classification.

The paper is organized as follows. In the Section 2, we recall the Riemann problem to the Euler equation and introduce some notations. We recall the arguments on the Cauchy problem in the Section 3, and summarize the recent results on the impermeable wall problem in the Section 4 . We study the outflow problem in the Section 5, and finally the inflow problem in the Section 6.

2. Riemann Problem. We recall the Riemann problem to the hyperbolic part of (1.1), that is, Euler equation. In what follows, we use the equivalent system to (1.1) which is described in terms of the specific volume $v(:=1 / \rho)$ and the velocity $u$ :

$$
\left\{\begin{array}{l}
\left(\frac{1}{v}\right)_{t}+\left(\frac{u}{v}\right)_{x}=0 \\
\left(\frac{u}{v}\right)_{t}+\left(\frac{u^{2}}{v}+p(v)\right)_{x}=\mu u_{x x}, \quad x>0, t>0 \\
p(v)=a v^{-\gamma}
\end{array}\right.
$$

Now we consider the Riemann problem on $\mathbf{R}$ to the hyperbolic part of (2.1) for given constant states $\left(v_{ \pm}, u_{ \pm}\right), v_{ \pm}>0$ :

$$
\begin{cases}\left(\frac{1}{v}\right)_{t}+\left(\frac{u}{v}\right)_{x}=0, & \\ \left(\frac{u}{v}\right)_{t}+\left(\frac{u^{2}}{v}+p(v)\right)_{x}=0, & x \in \mathbf{R}, t>0, \\ (v, u)(0, x)= \begin{cases}\left(v_{-}, u_{-}\right) & x<0 \\ \left(v_{+}, u_{+}\right) & x>0 .\end{cases} \end{cases}
$$

For smooth $v$ and $u$, the system (2.2) is rewritten in the form

$$
\left(\begin{array}{c}
v \\
u
\end{array}\right)_{t}+A(v, u)\left(\begin{array}{c}
v \\
u
\end{array}\right)_{x}=0
$$

where

$$
A(v, u)=\left(\begin{array}{cc}
u & -v \\
v p^{\prime}(v) & u
\end{array}\right)
$$

The eigenvalues $\left\{\lambda_{i}\right\}_{i=1}^{2}$ of $A(v, u)$ and corresponding right eigenvectors $\left\{r_{i}\right\}_{i=1}^{2}$ are given by

$$
\lambda_{i}(v, u)=u+v \lambda_{i}^{L}(v), \quad r_{i}(v)=\left(\begin{array}{c}
1 \\
-\lambda_{i}^{L}(v)
\end{array}\right)
$$

where $\lambda_{1}^{L}(v)=-\left|p^{\prime}(v)\right|^{1 / 2}$, and $\lambda_{2}^{L}(v)=\left|p^{\prime}(v)\right|^{1 / 2}$. We also define the sound speed $c(v)$ by

$$
c(v):=v\left|p^{\prime}(v)\right|^{\frac{1}{2}}=\sqrt{a \gamma} v^{-\frac{\gamma-1}{2}}
$$


Here note that $\lambda_{i}^{L}(v)$ are corresponding to the eigenvalues of the system in Lagrange coordinates setting (cf. (6.6)). Thanks to the properties $p^{\prime}(v)<0$ and $p^{\prime \prime}(v)>0$, the system (2.2) is strictly hyperbolic and its both characteristic fields are genuinely nonlinear for $v>0$. Let $\Omega=\left\{(v, u) \in \mathbf{R}_{+} \times \mathbf{R}\right\}$ be the phase space of $(v, u)$. In what follows, let us abbreviate $(v, u)$ to $w,\left(v_{ \pm}, u_{ \pm}\right)$to $w_{ \pm}$, and so on, for simplicity. Since the integral curves of the right eigenvectors are described by the ordinary differential equations $d u / d v=-\lambda_{i}^{L}(v)$ in $\Omega$, the rarefaction curves $R_{1}\left(w_{-}\right)$and $R_{2}\left(w_{-}\right)$for any fixed left state $w_{-} \in \Omega$ are defined by

$$
R_{i}\left(w_{-}\right)=\left\{w=(v, u) \in \Omega \mid u=u_{-}-\int_{v_{-}}^{v} \lambda_{i}^{L}(s) d s, u \geq u_{-}\right\} \quad i=1,2 .
$$

Similarly, for any fixed right state $w_{+} \in \Omega$, the rarefaction curves $\tilde{R}_{i}\left(w_{+}\right)$are defined. When $w_{+} \in R_{i}\left(w_{-}\right)(i=1,2)$, or equivalently $w_{-} \in \tilde{R}_{i}\left(w_{+}\right)$, the solution of the Riemann problem (2.2) consists of two constant states $w_{ \pm}$and one centered rarefaction wave which continuously connects the left and right states. We denote this solution by

$$
W_{i}^{R}\left(x / t ; w_{-}, w_{+}\right)=\left(V_{i}^{R}, U_{i}^{R}\right)\left(x / t ; w_{-}, w_{+}\right), \quad i=1,2,
$$

and simply call it $i$-rarefaction wave. On the other hand, if $w_{ \pm}$and $s \in \mathbf{R}$ satisfy the Rankine-Hugoniot condition

$$
\left\{\begin{array}{l}
-s\left(\frac{1}{v_{+}}-\frac{1}{v_{-}}\right)+\left(\frac{u_{+}}{v_{+}}-\frac{u_{-}}{v_{-}}\right)=0, \\
-s\left(\frac{u_{+}}{v_{+}}-\frac{u_{-}}{v_{-}}\right)+\left(\frac{u_{+}^{2}}{v_{+}}-\frac{u_{-}^{2}}{v_{-}}+p\left(v_{+}\right)-p\left(v_{-}\right)\right)=0,
\end{array}\right.
$$

then it is known the Riemann problem has a shock wave solution with the shock speed $s$. By elementary calculations to the R-H condition (2.5), the value $u_{+}$and the shock speed $s$ should be given in terms of $u_{-}$and $v_{ \pm}$by

$$
\left\{\begin{array}{l}
u_{+}=u_{-}-\left(v_{+}-v_{-}\right) s_{i}^{L}\left(v_{-}, v_{+}\right), \\
s=s_{i}\left(w_{-}, v_{+}\right):=u_{-}+v_{-} s_{i}^{L}\left(v_{-}, v_{+}\right), \quad i=1,2,
\end{array}\right.
$$

where

$$
s_{1}^{L}\left(v_{-}, v_{+}\right)=-\left|\frac{p\left(v_{+}\right)-p\left(v_{-}\right)}{v_{+}-v_{-}}\right|^{\frac{1}{2}}, s_{2}^{L}\left(v_{-}, v_{+}\right)=-s_{1}^{L}\left(v_{-}, v_{+}\right) .
$$

Combining the above together with the entropy condition

$$
\lambda_{i}\left(w_{+}\right)<s_{i}<\lambda_{i}\left(w_{-}\right),
$$

which is simply equivalent to $u_{+}<u_{-}$in our cases, we can define the shock curves $S_{1}\left(w_{-}\right)$and $S_{2}\left(w_{-}\right)$for any fixed left state $w_{-} \in \Omega$ by

$$
S_{i}\left(w_{-}\right)=\left\{w=(v, u) \in \Omega \mid u=u_{-}-\left(v-v_{-}\right) s_{i}^{L}\left(v_{-}, v\right), u \leq u_{-}\right\} .
$$

Similarly, for any fixed right state $w_{+} \in \Omega$, the shock curves $\tilde{S}_{i}\left(w_{+}\right)$are defined. When $w_{+} \in S_{i}\left(w_{-}\right)(i=1,2)$, or equivalently $w_{-} \in \tilde{S}_{i}\left(w_{+}\right)$, the solution of the Riemann problem (2.2) consists of two constant states $w_{ \pm}$and one shock discontinuity which 
connects the left and right states and propagates with the shock speed $s_{i}$. We denote this solution by

$$
W_{i}^{S}\left(x-s_{i} t ; w_{-}, w_{+}\right)=\left(V_{i}^{S}, U_{i}^{S}\right)\left(x-s_{i} t ; w_{-}, w_{+}\right), \quad i=1,2
$$

and simply call it $i$-shock wave.

For any fixed right state $w_{+} \in \Omega$, the state space $\Omega$ is divided into four domains by the rarefaction curves $\left\{R_{i}\left(w_{-}\right)\right\}_{i=1}^{2}$ and shock curves $\left\{S_{i}\left(w_{-}\right)\right\}_{i=1}^{2}$, that is, $R R\left(w_{-}\right)$ with the boundary $R_{1}\left(w_{-}\right)$and $R_{2}\left(w_{-}\right), R S\left(w_{-}\right)$with the boundary $R_{1}\left(w_{-}\right)$and $S_{2}\left(w_{-}\right), S R\left(w_{-}\right)$with the boundary $S_{1}\left(w_{-}\right)$and $R_{2}\left(w_{-}\right)$, and $S S\left(w_{-}\right)$with the boundary $S_{1}\left(w_{-}\right)$and $S_{2}\left(w_{-}\right)$. When $w_{+}$is located in one of the above four domains, the Riemann problem's solution is given by the combination of the corresponding 1and 2-waves. For example, if $w_{+} \in R S\left(w_{-}\right)$, then there exists a unique intermediate state $\bar{w} \in R_{1}\left(w_{-}\right)$satisfying $w_{+} \in S_{2}(\bar{w})$, and the solution is exactly given by

$$
W_{1}^{R}\left(x / t ; w_{-}, \bar{w}\right)+W_{2}^{S}\left(x-s_{2} t ; \bar{w}, w_{+}\right)-\bar{w} .
$$

Thus, all patterns of the solution of the Riemann problem are classified into eight cases depending on where $w_{+}$is located, that is, $\left\{R_{i}\left(w_{-}\right)\right\}_{i=1}^{2},\left\{S_{i}\left(w_{-}\right)\right\}_{i=1}^{2}, R R\left(w_{-}\right)$, $R S\left(w_{-}\right), S R\left(w_{-}\right)$, and $S S\left(w_{-}\right)$.

3. Viscous Shock Waves. We recall the viscous shock waves of (2.1) and the known results on the Cauchy problem and also the impermeable wall problem. The viscous shock wave $W$ with the shock speed $s \in \mathbf{R}$ is defined by special solution of (2.1) which has the form

$$
w(x, t)=(V, U)(\xi), \quad \xi=x-s t
$$

and smoothly connects the states $w_{ \pm} \in \Omega$ so that $W( \pm \infty)=w_{ \pm}$. Substituting (3.1) to $(2.1)$, we have the system of ordinary differential equations

$$
\left\{\begin{array}{l}
-s\left(\frac{1}{V}\right)^{\prime}+\left(\frac{U}{V}\right)^{\prime}=0, \\
-s\left(\frac{U}{V}\right)^{\prime}+\left(\frac{U^{2}}{V}+p(V)\right)^{\prime}=\mu U^{\prime \prime}, \quad \xi \in \mathbf{R} .
\end{array}\right.
$$

It is known that under the R-H condition (2.3) and the entropy condition (2.4), which implies $w_{+} \in S_{i}\left(w_{-}\right)(i=1$ or 2$)$, the equation is reduced to

$$
\left\{\begin{array}{l}
U(\xi)=u_{ \pm}-\left(V(\xi)-v_{ \pm}\right) s_{i}^{L}\left(v_{-}, v_{+}\right), \quad \xi=x-s_{i} t \\
\mu s_{i}^{L} V^{\prime}(\xi)=-p(V(\xi))+p\left(v_{ \pm}\right)-\left(V(\xi)-v_{ \pm}\right)\left(s_{i}^{L}\right)^{2}, \quad \xi \in \mathbf{R} \\
V( \pm \infty)=v_{ \pm}
\end{array}\right.
$$

where the shock speed $s=s_{i}\left(w_{-}, v_{+}\right)$is given by $u_{-}+v_{-} s_{i}^{L}\left(v_{-}, v_{+}\right)$. Due to the convexity of $p$, it is easy to show the system (3.3) has a unique solution up to shift. We denote this solution by

$$
W_{i}^{V S}\left(x-s_{i} t ; w_{-}, w_{+}\right)=\left(V_{i}^{V S}, U_{i}^{V S}\right)\left(x-s_{i} t ; w_{-}, w_{+}\right), \quad i=1,2,
$$

and simply call it $i$-viscous shock wave. 
Now we recall the results on the asymptotic behaviors of solutions of the initial value problem to the system (2.1) with the initial and far fields conditions

$$
\begin{gathered}
(v, u)(0, x)=\left(v_{0}, u_{0}\right)(x), \quad x \in \mathbf{R}, \\
\lim _{x \rightarrow \pm \infty}(v, u)(t, x)=\left(v_{ \pm}, u_{ \pm}\right), \quad t>0 .
\end{gathered}
$$

As we mentioned in the Section 1, the asymptotic sates are basically same as that of Riemann problem to the hyperbolic part, if only we replace the shock waves by the corresponding smooth viscous shock waves. Therefore, for any fixed $w_{-} \in \Omega$, depending on where $w_{+}$is located, the solution is expected to tend toward the following asymptotic states:

$$
\begin{array}{ll}
W_{i}^{R}\left(x / t ; w_{-}, w_{+}\right), & \text {for } w_{+} \in R_{i}\left(w_{-}\right), \\
W_{i}^{V S}\left(x-s_{i} t+\alpha_{i} ; w_{-}, w_{+}\right), & \text {for } w_{+} \in S_{i}\left(w_{-}\right), \\
W_{1}^{R}\left(x / t ; w_{-}, \bar{w}\right)+W_{2}^{R}\left(x / t ; \bar{w}, w_{+}\right)-\bar{w}, & \text { for } w_{+} \in R R\left(w_{-}\right), \\
W_{1}^{V S}\left(x-s_{1} t+\alpha_{1} ; w_{-}, \bar{w}\right)+ & \\
W_{2}^{V S}\left(x-s_{2} t+\alpha_{2} ; \bar{w}, w_{+}\right)-\bar{w}, & \text { for } w_{+} \in S S\left(w_{-}\right), \\
W_{1}^{V S}\left(x-s_{1} t+\alpha_{1} ; w_{-}, \bar{w}\right)+W_{2}^{R}\left(x / t ; \bar{w}, w_{+}\right)-\bar{w}, & \text { for } w_{+} \in S R\left(w_{-}\right), \\
W_{1}^{R}\left(x / t ; w_{-}, \bar{w}\right)+W_{2}^{V S}\left(x-s_{2} t+\alpha_{2} ; \bar{w}, w_{+}\right)-\bar{w}, & \text { for } w_{+} \in R S\left(w_{-}\right) .
\end{array}
$$

As for these asymptotic behaviors, there have been many works where all results are stated for the equivalent problem to (1.1) described in the Lagrangian mass coordinates, in which the system can be handled in easier way than that in the Eulerian coordinates. Here let us make a survey in terms of the Lagrangian coordinates. Matsumura \& Nishihara [9] first treated the case $w_{+} \in S_{i}\left(w_{-}\right)$and succeeded in showing that if $(\gamma-1)\left|w_{+}-w_{-}\right|$is suitably small, the viscous shock wave $W_{i}^{V S}\left(x-s_{i} t ; w_{-}, w_{+}\right)$ is asymptotically stable for small initial perturbations with zero integrals, so that the phase shift $\alpha$ does not occur. For the initial data with non-zero integrals, Liu [2][3] studied the criterion how to determine the phase shift based on his deep insight, and Szepessy \& Xin [14] succeeded in showing, for the system with artificial viscosity terms which make the system uniformly parabolic, that if $\left|w_{+}-w_{-}\right|$and the initial perturbations are suitably small, then the solution tends toward the $W_{i}^{V S}\left(x-s_{i} t+\alpha ; w_{-}, w_{+}\right)$ where the phase shift $\alpha$ is uniquely determined by the Liu's criterion. Although the introduction in [14] reads the physical viscosity case can be similarly treated, it seems not to be so trivial. The arguments by Szepessy [15] on the asymptotic stability of the viscous shock wave to the Broadwell model are believed enough to compensate the gap. However the complete proof for our original system is not available in any publications up to now. Therefore we really hope to give a complete and simpler proof to our physical viscosity case, even if we use a special feature of our system. In the case $w_{+} \in S S\left(w_{-}\right)$, combining the arguments in [9] together with that in [2][3], we can show as a kind of exercise that if $\left|w_{+}-w_{-}\right|$is suitably small and the initial data is suitably close to $W_{1}^{V S}\left(x ; w_{-}, \bar{w}\right)+W_{2}^{V S}\left(x ; \bar{w}, w_{+}\right)-\bar{w}$, then the solution tends toward

$$
W_{1}^{V S}\left(x-s_{1} t+\alpha_{1} ; w_{-}, \bar{w}\right)+W_{2}^{V S}\left(x-s_{2} t+\alpha_{2} ; \bar{w}, w_{+}\right)-\bar{w}
$$


where the shifts $\alpha_{1}$ and $\alpha_{2}$ are uniquely determined by the initial data. In the cases $w_{+} \in\left(R_{1} \cup R_{2} \cup R R\right)\left(w_{-}\right)$, Matsumura and Nishihara [10][11][12] succeeded in obtaining the complete results that the asymptotic state is given by the corresponding either simple rarefaction wave or combination of two rarefaction waves of the Euler equation without any smallness conditions on $\left|w_{+}-w_{-}\right|, \gamma$, and the initial data. Finally we should emphasize the cases $w_{+} \in(S R \cup R S)\left(w_{-}\right)$are still entirely open. These cases would be very interesting and challenging, because we could expect the interaction between the tails of the rarefaction and viscous shock waves are persistent and subtle enough to make controlling the phase shift of the viscous shock very entangled.

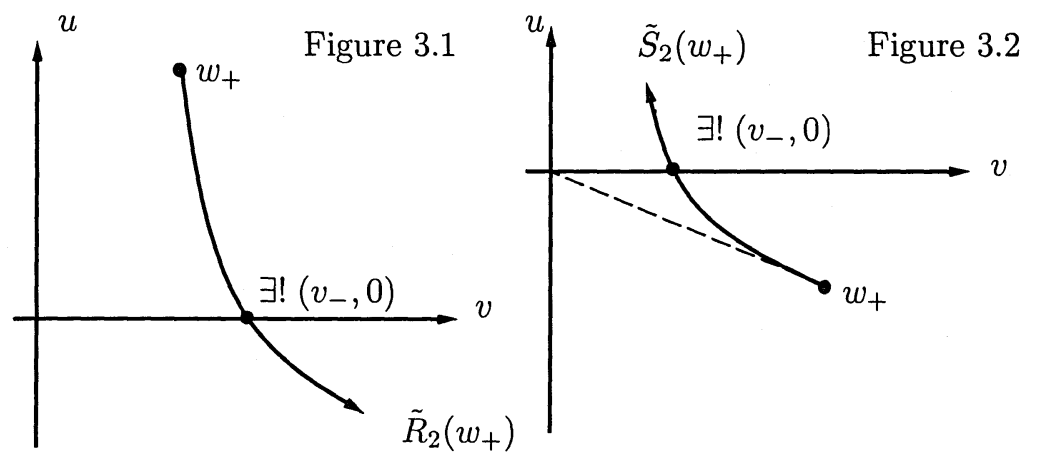

Let us turn to the case of the impermeable wall problem $(1.1) \sim(1.4)_{1}$. Following our criterion how to expect the asymptotic state as mentioned in the introduction, let us consider the corresponding Riemann problem (2.2) where the left state $v_{-}$is freely given, that is, all candidates of the left states $w_{-}$form the $v$-axis in $\Omega$. When $u_{+}>0$, as the Figure 3.1 shows, we can see that the Riemann solution includes an incoming wave which is not consistent with the boundary condition $(1.4)_{1}$ for all $w_{-}$on the $v$ axis except the unique point such that $\left(v_{-}, 0\right) \in \tilde{R}_{2}\left(w_{+}\right)$, equivalently $w_{+} \in R_{2}\left(v_{-}, 0\right)$. For this $v_{-}$, the Riemann solution consists of only an outgoing rarefaction wave $W_{2}^{R}\left(x / t ; w_{-}, w_{+}\right)$, and we do expect it as the asymptotic state of the solution. In fact, this conjecture was completely proved by Matsumura \& Nishihara [12] without any smallness conditions on $\left|w_{+}-w_{-}\right|, \gamma$, and the initial data. Similarly when $u_{+}<0$, as the Figure 3.2 shows, we can see that $\left(v_{-}, 0\right) \in \tilde{S}_{2}\left(w_{+}\right)$is the only the option for which the Riemann solution is consistent with the boundary condition, and thus we expect the asymptotic state to be the corresponding viscous shock wave $W_{2}^{V S}\left(x-s_{2} t+\alpha ; w_{-}, w_{+}\right)$with a proper phase shift $\alpha$. Here we should note the shock speed $s_{2}$ is positive no matter how negatively large $u_{+}$is. (This fact is showed as follows: For a shock wave with the zero shock speed $s$, the left state $w_{-}$turns out to be located on the straight line connecting the origin and the right state $w_{+}$, due to the first equation of the R-H condition (2.5). This implies the shock wave has a positive shock speed $s_{2}$ if $w_{-} \in \tilde{S}_{2}\left(w_{+}\right)$is located above the straight line, and has the negative speed $s_{2}$ if below the line (cf. Figure 3.2).) Recently Matsumura \& Mei [8] succeeded in obtaining a positive result on this conjecture that if the viscous shock wave is suitably far away from the boundary at the initial time and if the initial perturbations are small enough, then the solution tends toward a properly shifted viscous shock wave whose phase shift is uniquely and explicitly determined by the initial perturbations. Thus, the asymptotic behaviors of the solutions for the impermeable wall problem can be basically classified into only two cases, whether $u_{+}$ is positive or negative, in contrast with the Cauchy problem, eight cases.

Finally in this section, we should make some remarks on the phase shift just 
mentioned above to compare our case with that of the scalar viscous conservation laws, whose IBVP with Dirichlet zero boundary condition were studied in Liu \& Yu [6], and Liu \& Nishihara [5]. Even in these scalar cases, in order to locate the phase shift, they needed the laborious analysis (pointwise estimates via Green function in [6], technical weighted estimates in [5]), since the shift can't be determined explicitly because of the viscosity term. So we had thought our system case is much more difficult in many aspects. However it turns out that our system with physical viscosity on the half space has several better features than those both for the scalar cases with boundary and also for the systems without boundary. In particular, our system is not uniformly parabolic, i.e., there is no viscosity term for the density, and we can't impose the boundary value of the density, which usually gives various difficulties. This is really the reason why we can specify the phase shift $\alpha$ of $W_{2}^{V S}\left(x-s_{2} t+\alpha\right)$ only by the hyperbolic equation for the density, and we can expect that value of $v(0, t)$ on the boundary is automatically controlled to tend to the value $v_{-}$by the structure of the system itself so that the whole solution $w(x, t)$ tends to $W_{2}^{V S}\left(x-s_{2} t+\alpha\right)$ with the same $\alpha$. Let us show how to specify the shift $\alpha$ more precisely. Let denote $W_{2}^{V S}\left(x-s_{2} t+\alpha ; w_{-}, w_{+}\right)$simply by $W(x-s t+\alpha)$. Then, by the equation of the conservation of density, we deduce

$$
\left(\frac{1}{v}-\frac{1}{V}\right)_{t}+\left(\frac{u}{v}-\frac{U}{V}\right)_{x}=0 .
$$

Integrating (3.5) with respect to both $x$ and $t$, we have

$$
\int_{0}^{\infty} \frac{1}{v}-\frac{1}{V} d x=\int_{0}^{\infty} \frac{1}{v_{0}(x)}-\frac{1}{V(x+\alpha)} d x-\int_{0}^{t} \frac{U(-s \tau+\alpha)}{V(-s \tau+\alpha)} d \tau .
$$

If we assume that $v-V$ tends to zero well enough, the right hand side of (3.6) should satisfy

$$
\int_{0}^{\infty} \frac{1}{v_{0}(x)}-\frac{1}{V(x+\alpha)} d x-\int_{0}^{\infty} \frac{U(-s \tau+\alpha)}{V(-s \tau+\alpha)} d \tau=0
$$

Set the left hand side of (3.7) by $I(\alpha)$ and differentiate it with respect to $\alpha$ as

$$
\frac{d I(\alpha)}{d \alpha}=-\frac{1}{v_{+}}-\frac{1}{s}\left(-s \frac{1}{V(\alpha)}+\frac{U(\alpha)}{V(\alpha)}\right) .
$$

Since the first equation of (3.2) gives

$$
-s \frac{1}{V(\alpha)}+\frac{U(\alpha)}{V(\alpha)}=-s \frac{1}{v_{-}}, \quad \alpha \in \mathbf{R}^{1},
$$

we deduce from (3.8) and (3.9) that

$$
I(\alpha)=-\left(\frac{1}{v_{+}}-\frac{1}{v_{+}}\right) \alpha+I(0) .
$$

Thus it turns out that the phase shift $\alpha$ should be given by the formula

$$
\alpha=\frac{1}{\rho_{+}-\rho_{-}}\left\{\int_{0}^{\infty} \frac{1}{v_{0}(x)}-\frac{1}{V(x)} d x-\int_{0}^{\infty} \frac{U(-s \tau)}{V(-s \tau)} d \tau\right\}
$$


4. Boundary Layers. Now we are ready to start to consider the Inflow and Outflow Problems. As mentioned in the introduction, the classification of the large time asymptotic states are expected to depend crucially on whether the state either on the boundary and at far field is subsonic, transonic, or supersonic. Hence we divide the domain $\Omega$ into 5 regions (see Figure 4.1)

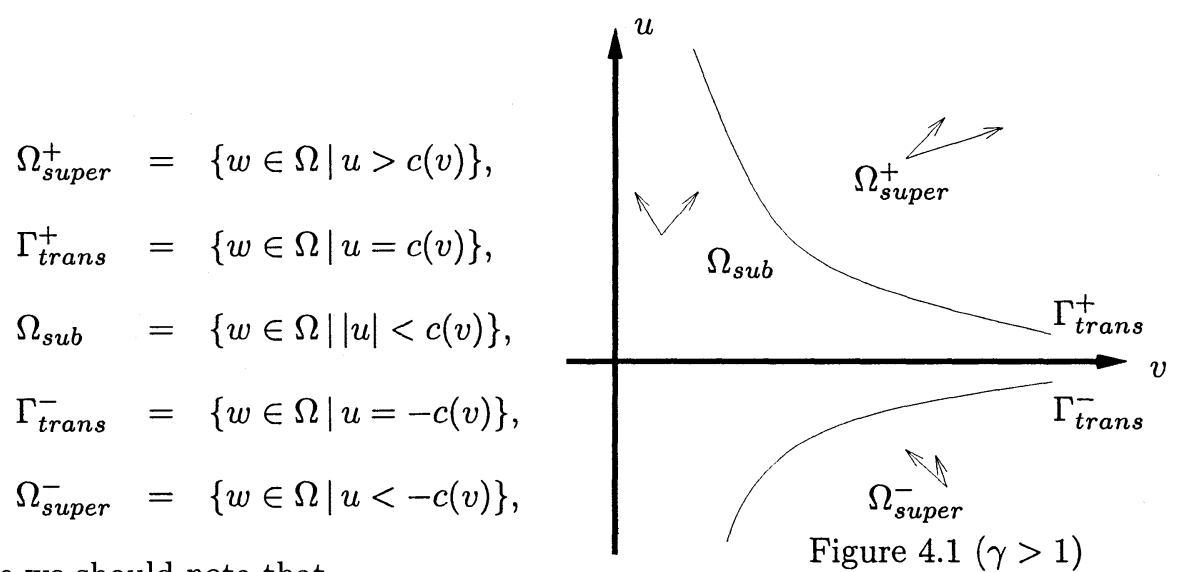

where we should note that

Figure $4.1(\gamma>1)$

$$
\begin{array}{lll}
w \in \Omega_{\text {super }}^{+} & \rightarrow \quad 0<\lambda_{1}(w)<\lambda_{2}(w), \\
w \in \Gamma_{\text {trans }}^{+} & \rightarrow \quad 0=\lambda_{1}(w)<\lambda_{2}(w), \\
w \in \Omega_{\text {sub }} & \rightarrow \quad \lambda_{1}(w)<0<\lambda_{2}(w), \\
w \in \Gamma_{\text {trans }}^{-} & \rightarrow \quad \lambda_{1}(w)<\lambda_{2}(w)=0, \\
w \in \Omega_{\text {super }}^{-} & \rightarrow \quad \lambda_{1}(w)<\lambda_{2}(w)<0 .
\end{array}
$$

Now let us pick up an example from the Outflow Problem(1.1) $(1.4)_{2}$ which show that any corresponding Riemann solution is not consistent with the boundary condition $(1.4)_{2}$. For fixed $u_{-}<0, v_{+}>0$, and $u_{+}>0$, consider the Riemann problem with free $v_{-}$as in the previous sections.

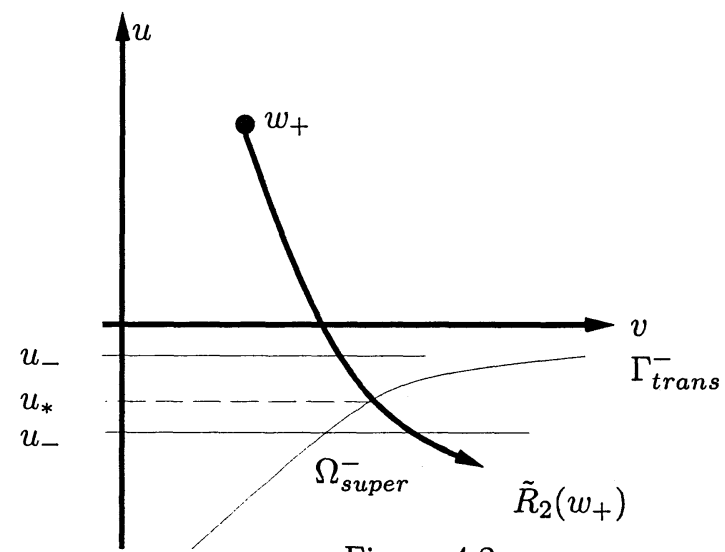

Figure 4.2 
As the Figure 4.2 shows, 2-rarefacton curve $\tilde{R}_{2}\left(w_{+}\right)$intersects $\Gamma_{\text {trans }}^{-}$at a point denoted by $w_{*}=\left(v_{*}, u_{*}\right)$. If $u_{*} \leq u_{-}<0$, as in the impermeable wall problem, there exist a unique $v_{-} \in \tilde{R}_{2}\left(w_{+}\right)$so that we can expect the solution tend toward $W_{2}^{R}\left(x / t ; w_{-}, w_{+}\right)$and no boundary layer appear. However, if $u_{-}<u_{*}$ then, even for the most plausible option $v_{-} \in \tilde{R}_{2}\left(w_{+}\right)$, the Riemann solution $W_{2}^{R}\left(x / t ; w_{-}, w_{+}\right)$ includes a part $W_{2}^{R}\left(x / t ; w_{-}, w_{*}\right)$ which goes into the boundary, so is not consistent with the boundary condition $(1.4)_{2}$. In this situation, we do expect the interaction between the incoming wave and the boundary condition select a new $v_{-}$and form a boundary layer solution which connects $w_{-}$and $w_{*}$. In this paper, we refer to stationary solution $W(x)$ of $(1.1)$ with the conditions $W(0)=w_{-}$and $W(+\infty)=w_{+}$as boundary layer solution connecting $w_{-}$and $w_{+}$, and denote it by

$$
W^{B L}\left(x ; w_{-}, w_{+}\right)=\left(V^{B L}\left(x ; w_{-}, w_{+}\right), U^{B L}\left(x ; w_{-}, w_{+}\right)\right)
$$

More precisely, the boundary layer $W^{B L}\left(x ; w_{-}, w_{+}\right)$is defined by the solution of

$$
\left\{\begin{array}{l}
\left(\frac{U}{V}\right)^{\prime}=0, \\
\left(\frac{U^{2}}{V}+p(V)\right)^{\prime}=\mu U^{\prime \prime}, \quad x>0 \\
W(0)=w_{-}, \quad W(+\infty)=w_{+} .
\end{array}\right.
$$

Let us investigate the existence of $W(x)$ and its properties. We first consider the case corresponding to the Outflow Problem, that is, $u_{-}<0$ (the following arguments on stationary solution are basically due to Kawashima \& Nishibata[1]). The first equation of (4.1) immediately implies

$$
\frac{U(x)}{V(x)}=\frac{u_{+}}{v_{+}}=\frac{u_{-}}{v_{-}}, \quad x>0
$$

in particular, that for a fixed $w_{+}$, the boundary state $w_{-}$should be located on the line $B L\left(w_{+}\right)$which passes through the origin and $w_{+}$,

$$
B L\left(w_{+}\right)=\left\{w \in \Omega \mid u=-s_{0} v, s_{0}=-\frac{u_{+}}{v_{+}}\right\}
$$

Integrating the second equation of (4.1) with the aid of (4.2), we have

$$
\left\{\begin{array}{l}
s_{0} \mu V^{\prime}=-s_{0}^{2}\left(V-v_{+}\right)+p\left(v_{+}\right)-p(V), \quad x>0 \\
V(0)=v_{-}, \quad V(+\infty)=v_{+}
\end{array}\right.
$$

Here note that $s_{0}=-u_{ \pm} / v_{ \pm}>0$. We can check whether $V^{\prime}$ has right sign or not on the interval between $v_{-}$and $v_{+}$in (4.3) as follows (refer to Figure 4.3). 
First fix $w_{+}$, then draw the line $B L\left(w_{+}\right)$which always intersects the transonic line $\Gamma_{\text {trans }}^{-}, u / v=$ $-\left|p^{\prime}(v)\right|^{1 / 2}$. Denote the intersection point by $w_{*}=\left(v_{*}, u_{*}\right)$. Consider the graph of $p(v)$ and draw the tangential line at $\left(v_{*}, p\left(v_{*}\right)\right)$. It easily turns out that the slope of the tangential line exactly equals to $-s_{0}^{2}$. Then draw the line with the same slope $-s_{0}^{2}$ which passes through the point $\left(v_{+}, p\left(v_{+}\right)\right)$, denote this line by $l\left(w_{+}\right)=\{(v, q(v))\}$. Note $q(v)$ exactly equals to $-s_{0}^{2}\left(v-v_{+}\right)+p\left(v_{+}\right)$. Thus for any $w_{-} \in B L\left(w_{+}\right)$, we can easily check the sign of $V^{\prime}$ on the interval between $v_{-}$and $v_{+}$in (4.3) by seeing how the two graphs of $p(v)$ and $q(v)$ intersect. If $w_{+} \in \Omega_{\text {super }}^{-}$, we can see that the 2 -shock curve $\tilde{S}_{2}\left(w_{+}\right)$intersects the line $B L\left(w_{+}\right)$ at not only $w_{+}$but another point, be denoted by $\tilde{w}=(\tilde{v}, \tilde{u})$, correspondingly the line $l\left(w_{+}\right)$intersects the graph of $p(v)$ at not only $v=v_{+}$ but $v=\tilde{v}$. Then it easily turns out

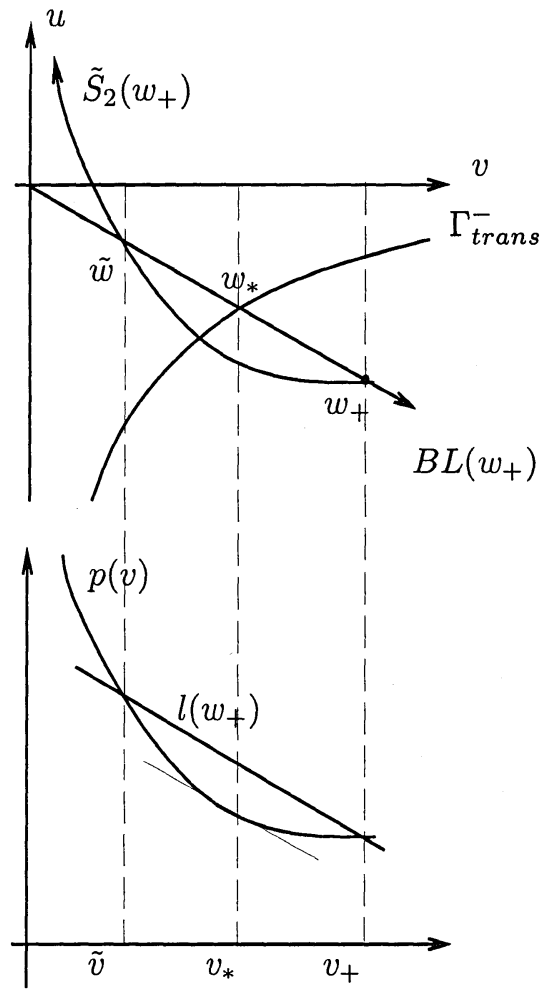

Figure 4.3

that $V^{\prime}$ has a right sign for $u_{-}<\tilde{u}$, that is, there exists the boundary layer solution $W^{B L}\left(x ; w_{-}, w_{+}\right)$, and have a wrong sign for $0>u_{-}>\tilde{u}$. As for $u_{-}=\tilde{u}$, although the sign is right, there exists no boundary layer solution but the stationary viscous shock wave $W_{2}^{S}\left(x+\alpha ; w_{-}, w_{+}\right)$. If $w_{+} \in \Gamma_{\text {trans }}^{-}$, we can also see that the 2 -shock curve $\tilde{S}_{2}\left(w_{+}\right)$tangentially intersects the line $B L\left(w_{+}\right)$at $w_{+}$and has no other intersection points. Then $V^{\prime}$ has a right sign only for $u_{-}<u_{+}$, and the boundary layer solution tends toward $w_{+}$at the algeblaic rate $O(1 / x)$ as $x$ goes to $+\infty$, because the line $l\left(w_{+}\right)$coincides with the tangential line of the graph of $p(v)$ at $v=v_{*}=v_{+}$(then we say the boundary layer solution is "degenerate"). Thus by these arguments above, we have the following

Proposition 4.1. Suppose $u_{+}<0$ and $w_{-} \in B L\left(w_{+}\right)$.

i) If $w_{+} \in \Omega_{\text {super }}^{-}$and $0>u_{-} \geq \tilde{u}$, then there exists no boundary layer solution.

ii) If $w_{+} \in \Omega_{\text {super }}^{-}$and $\tilde{u}>u_{-}>u_{+}$, then there exists a unique boundary layer solution of (4.1) and positive constants $C$ and $\delta$ such that

$$
V^{\prime}(x)>0, \quad x>0 \text {, and }\left|W(x)-w_{+}\right| \leq C \exp (-\delta x), x \rightarrow+\infty .
$$

iii) If $w_{+} \in \Omega_{\text {super }}^{-}$and $u_{+}>u_{-}$, then there exists a unique boundary layer solution of (4.1) and positive constants $C$ and $\delta$ such that

$$
V^{\prime}(x)<0, \quad x>0, \text { and }\left|W(x)-w_{+}\right| \leq C \exp (-\delta x), x \rightarrow+\infty .
$$

iv) If $w_{+} \in \Gamma_{\text {trans }}^{-}$and $0>u_{-} \geq u_{+}$, then there exists no boundary layer solution. 
v) If $w_{+} \in \Gamma_{\text {trans }}^{-}$and $u_{+}>u_{-}$, then there exists a unique degenerate boundary layer solution of (4.1) and a positive constants $C$ such that

$$
V^{\prime}(x)<0, \quad x>0, \text { and } \quad\left|W(x)-w_{+}\right| \leq \frac{C}{x}, x \rightarrow+\infty
$$

vi) If $w_{+} \in \Omega_{\text {sub }}^{-}$, then there exists no boundary layer solution.

Next we consider the boundary layer solution which satisfy $(4.2)(4.3)$ corresponding to the Inflow Problem, that is, $u_{-}>0$. In this case, note $s_{0}=-u_{ \pm} / v_{ \pm}<0$, and fix $w_{-}\left(u_{-}>0\right)$ at first for later arguments. As in the prvious case, draw the line

$$
B L\left(w_{-}\right)=\left\{w \in \Omega \mid u=-s_{0} v, s_{0}=-\frac{u_{-}}{v_{-}}\right\}
$$

which always intersects the transonic line $\Gamma_{\text {trans }}^{+}, u / v=\left|p^{\prime}(v)\right|^{1 / 2}$, denote the intersection point by $w_{*}=\left(v_{*}, u_{*}\right)$, and then check the sign of $V^{\prime}$ in (4.3) for any $w_{+} \in B L\left(w_{-}\right)$. In particular, if $w_{\in} \Omega_{\text {super }}^{+}$, the 1 -shock curve $S_{1}\left(w_{-}\right)$intersects the line $B L\left(w_{-}\right)$whose intersection point is denoted by $\tilde{w}$, and $V^{\prime}$ has a right sign only for $0<u_{+}<\tilde{u}$.

Proposition 4.2. Suppose $u_{-}>0$ and $w_{+} \in B L\left(w_{-}\right)$.

i) If $w_{-} \in \Omega_{\text {sub }} \cup \Gamma_{\text {trans }}^{+}$and $0<u_{+}<u_{-}$, then there exists a unique boundary layer solution of (4.1) and positive constants $C$ and $\delta$ such that

$$
V^{\prime}(x)<0, \quad x>0, \text { and }\left|W(x)-w_{+}\right| \leq C \exp (-\delta x), x \rightarrow+\infty .
$$

ii) If $w_{-} \in \Omega_{s u b}$ and $u_{-}<u_{+}<u_{*}$, then there exists a unique boundary layer solution of (4.1) and positive constants $C$ and $\delta$ such that

$$
V^{\prime}(x)>0, \quad x>0, \text { and }\left|W(x)-w_{+}\right| \leq C \exp (-\delta x), x \rightarrow+\infty .
$$

iii) If $w_{-} \in \Omega_{\text {sub }}$ and $u_{+}=u_{*}$, then there exists a unique degenerate boundary layer solution of (4.1) and positive constants $C$ and $\delta$ such that

$$
V^{\prime}(x)>0, \quad x>0, \text { and }\left|W(x)-w_{+}\right| \leq \frac{C}{x}, x \rightarrow+\infty .
$$

iv) If $w_{-} \in \Omega_{\text {sub }} \cup \Gamma_{\text {trans }}^{+}$and $u_{*}<u_{+}$, then there exists no boundary layer solution. v) If $w_{-} \in \Omega_{\text {super }}^{+}$and $0<u_{+}<\tilde{u}$, then there exists a unique boundary layer solution of (4.1) and positive constants $C$ and $\delta$ such that

$$
V^{\prime}(x)<0, \quad x>0, \text { and }\left|W(x)-w_{+}\right| \leq C \exp (-\delta x), x \rightarrow+\infty .
$$

vi) If $w_{-} \in \Omega_{\text {super }}^{+}$and $\tilde{u} \leq u_{+}$, then there exists no boundary layer solution.

5. Outflow Problem. In this section, using the arguments in the previous sections, we try to classify all asymptotic behaviors of solutions of the Outflow Problem $(1.1) \sim(1.4)_{2}$. We primarily divide into three cases depending on where $w_{+}$is located. 
(i)

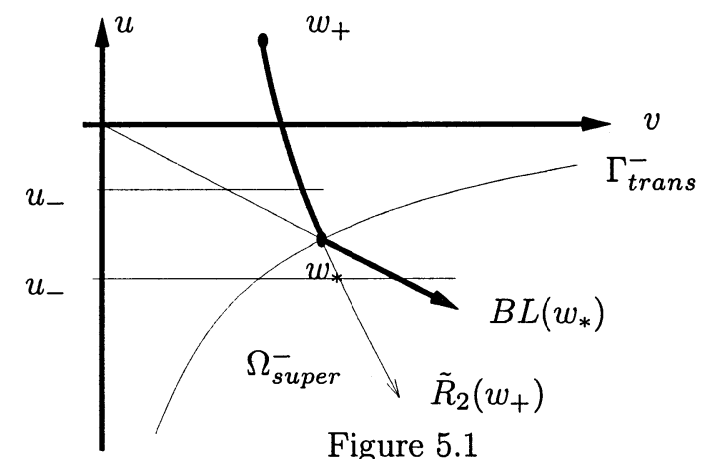

(i) $0>u_{-} \geq u_{*}$ : There exists a unique $v_{-}$such that $w_{-} \in \tilde{R}_{2}\left(w_{+}\right)$and the asymptotic state is expected to be

$$
W_{2}^{R}\left(x / t ; w_{-}, w_{+}\right)
$$

(ii) $u_{*}>u_{-}$: There exists a unique $v_{-}$such that $w_{-} \in \tilde{B L_{2}}\left(w_{*}\right)$ and the asymptotic state is expected to be

$$
W^{B L}\left(x ; w_{-}, w_{*}\right)+W_{2}^{R}\left(x / t ; w_{*}, w_{+}\right)-w_{*} .
$$

where note that the boundary layer solution $W^{B L}\left(x ; w_{-}, w_{*}\right)$ is degenerate.

- $u_{+}<0, w_{+} \in \Omega_{s u b} \cup \Gamma_{\text {trans }}^{-}$(see Figure 5.2):

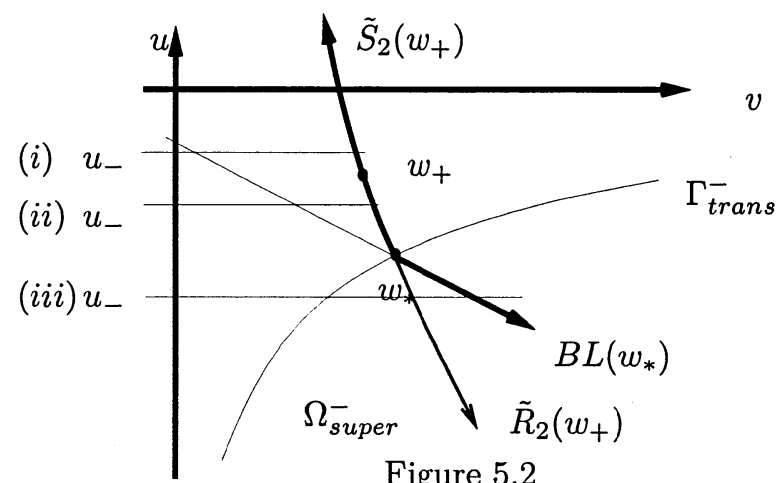

Figure 5.2

(i) $0>u_{-}>u_{+}:$There exists a unique $v_{-}$such that $w_{-} \in \tilde{S}_{2}\left(w_{+}\right)$and the asymptotic state is expected to be

$$
W_{2}^{V S}\left(x-s_{2} t+\alpha ; w_{-}, w_{+}\right) \text {for some } \alpha .
$$

(ii) $u_{+}>u_{-} \geq u_{*}$ : There exists a unique $v_{-}$such that $w_{-} \in \tilde{R}_{2}\left(w_{+}\right)$and the asymptotic state is expected to be

$$
W_{2}^{R}\left(x / t ; w_{-}, w_{+}\right) .
$$


(iii) $u_{*}>u_{-}:$There exists a unique $v_{-}$such that $w_{-} \in \tilde{B L_{2}}\left(w_{*}\right)$ and the asymptotic state is expected to be

$$
W^{B L}\left(x ; w_{-}, w_{*}\right)+W_{2}^{R}\left(x / t ; w_{*}, w_{+}\right)-w_{*} .
$$

where note that the boundary layer solution $W^{B L}\left(x ; w_{-}, w_{*}\right)$ is degenerate.

$\bullet u_{+}<0, w_{+} \in \Omega_{\text {super }}^{-}$(see Figure 5.3):

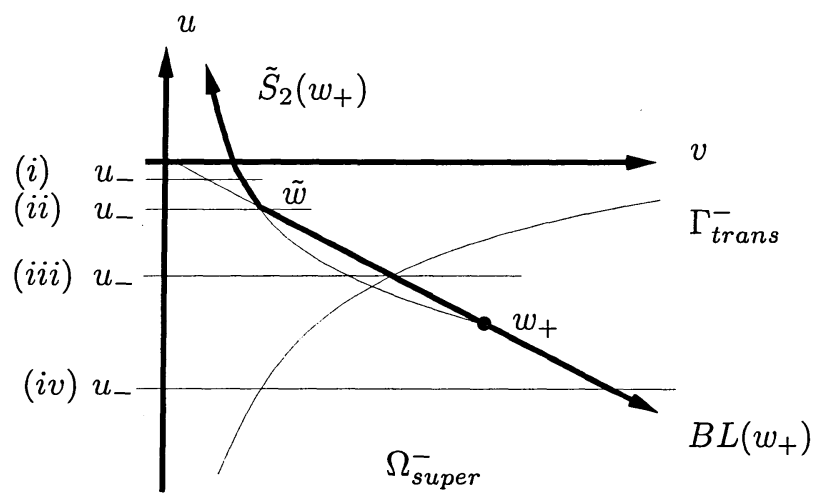

Figure 5.3

(i) $0>u_{-}>\tilde{u}$ : There exists a unique $v_{-}$such that $w_{-} \in \tilde{S}_{2}\left(w_{+}\right)$and the asymptotic state is expected to be

$$
W_{2}^{V S}\left(x-s_{2} t+\alpha ; w_{-}, w_{+}\right) \text {for some } \alpha .
$$

(ii) $u_{-}=\tilde{u}$ : There exists a unique $v_{-}$such that $w_{-} \in \tilde{S}_{2}\left(w_{+}\right)$and the asymptotic state is expected to be

$$
W_{2}^{V S}\left(x+\alpha(t) ; w_{-}, w_{+}\right) \text {for some } \alpha(t) \nearrow+\infty \text {. }
$$

(iii) $\tilde{u}>u_{-}>u_{+}$: There exists a unique $v_{-}$such that $w_{-} \in \tilde{B L_{2}}\left(w_{+}\right)$and the asymptotic state is expected to be

$$
W^{B L}\left(x ; w_{-}, w_{+}\right) \text {with }\left(V^{B L}\right)^{\prime}<0 .
$$

(iv) $u_{+}>u_{-}$: There exists a unique $v_{-}$such that $w_{-} \in B L\left(w_{+}\right)$and the asymptotic state is expected to be

$$
W^{B L}\left(x ; w_{-}, w_{+}\right) \text {with }\left(V^{B L}\right)^{\prime}>0 .
$$

Among above cases, let us make some comments on the behavior (5.7) which is subtle and interesting. In this case, the corresponding viscous shock wave $W_{2}^{V S}\left(x ; w_{-}, w_{+}\right)$ is stationary, so there is always a gap between the value $w_{-}$and $W_{2}^{V S}\left(0+\alpha ; w_{-}, w_{+}\right)$ on the boundary for any constant shift $\alpha$, which makes us expect the shift $\alpha$ rather depend on $t$ and increase up to $+\infty$ as $t \rightarrow+\infty$ in order for the gap to decrease to zero. This case is just corresponding to that for the Burgers equation in Liu \& $\mathrm{Yu}$ [6].

Only the results rigorously proved concerning above asymptotic behaviors are given by Kawashima \& Nishibata [1]. They proved that if $w_{+} \in \Omega_{\text {super }}^{-}, w_{-} \in$ 
$B L\left(w_{+}\right)$, and $\left|w_{+}-w_{-}\right|$is suitably small, then the boundary layer solution $W^{B L}\left(x ; w_{-}, w_{+}\right)$is asymptotically stable. It should be noted that they employ a method where the monotonicity of the boundary layer solution is not used, so they can treat the both cases $w_{-}<w_{+}$and $w_{+}<w_{-}$(see [1] for details). All other cases are open problems! Finally in this section, we should point out a difficulty the Outflow Problem faces. If we employ, so called, the Lagrangian mass coordinates system, which usually makes the form of equations simpler and the treatment of the equations easier, the problem becomes a free boundary value problem which makes the treatment of boundary more difficult. On the other hand, as we will see in the next section, the Inflow Problem become a corresponding IBVP with a prescribed moving boundary $\left(x=s_{0} t, s_{0}=-u_{-} / v_{-}\right)$in the Lagrangian mass coordinates, since the both values of velocity and density on the boundary are given.

6. Inflow Problem. In this section, we try to classify all asymptotic behaviors of solutions of the Inflow Problem $(1.1) \sim(1.4)_{3}$, and show some cases are rigorously proved. Thanks to all arguments in the Section 3 and 4 , we primarily classify into two cases in terms of the location of $w_{-}$, that is, $w_{-} \in \Omega_{\text {sub }} \cup \Gamma_{\text {trans }}^{+}$or $w_{-} \in \Omega_{\text {super }}^{+}$, and then for each $w_{-}$classify into many sub-cases depending on where $w_{+}$is located. For $w_{-} \in \Omega_{s u b} \cup \Gamma_{\text {trans }}^{+}$, we basically divide the phase space $\Omega$ of $w_{+}$into 13 regions, and for $w_{-} \in \Omega_{\text {super }}^{+}, 14$ regions.

- $w_{-} \in \Omega_{\text {sub }} \cup \Gamma_{\text {trans }}^{+} \quad$ (see Figure 6.1):

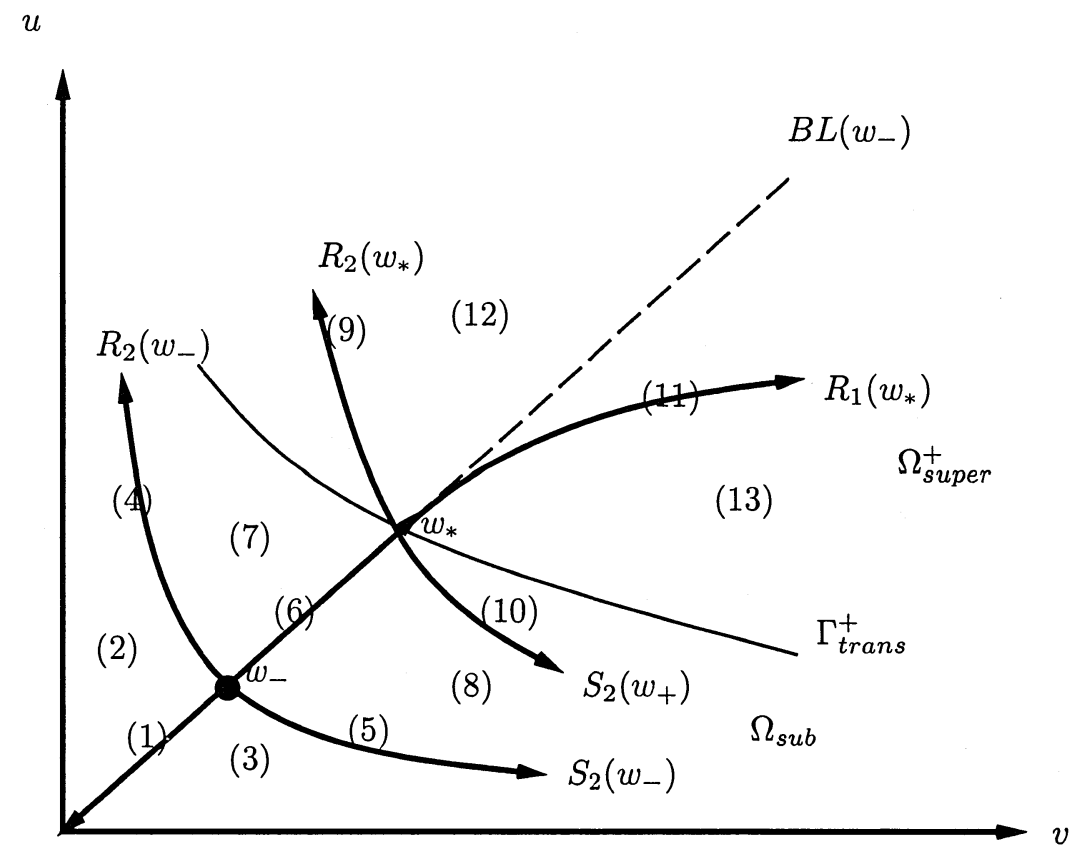

Figure 6.1

(1) $w_{+} \in B L\left(w_{-}\right), 0<u_{+}<u_{-}$: The asymptotic state is expected to be $W^{B L}\left(x ; w_{-}, w_{+}\right)$ 
(2) $w_{+} \in \operatorname{Region}(2):$ There exists a unique $\bar{w} \in B L\left(w_{-}\right)\left(\bar{u}<u_{-}\right)$such that $w_{+} \in$ $R_{2}(\bar{w})$ and the asymptotic state is expected to be

$$
W^{B L}\left(x ; w_{-}, \bar{w}\right)+W_{2}^{R}\left(x / t ; \bar{w}, w_{+}\right)-\bar{w} .
$$

(3) $w_{+} \in \operatorname{Region}(3):$ There exists a unique $\bar{w} \in B L\left(w_{-}\right)\left(\bar{u}<u_{-}\right)$such that $w_{+} \in$ $S_{2}(\bar{w})$ and the asymptotic state is expected to be

$$
W^{B L}\left(x ; w_{-}, \bar{w}\right)+W_{2}^{V S}\left(x-s_{2} t+\alpha ; \bar{w}, w_{+}\right)-\bar{w} .
$$

(4) $w_{+} \in R_{2}\left(w_{-}\right)$: The asymptotic state is expected to be

$$
W_{2}^{R}\left(x / t ; w_{-}, w_{+}\right) .
$$

(5) $w_{+} \in S_{2}\left(w_{-}\right)$: The asymptotic state is expected to be

$$
W_{2}^{V S}\left(x-s_{2} t+\alpha ; w_{-}, w_{+}\right)
$$

(6) $w_{+} \in B L\left(w_{-}\right), u_{-}<u_{+} \leq u_{*}$ : The asymptotic state is expected to be

$$
W^{B L}\left(x ; w_{-}, w_{+}\right)
$$

where note that if $w_{+}=w_{*}$, then the boundary layer $W^{B L}\left(x ; w_{-}, w_{+}\right)$is degenerate.

(7) $w_{+} \in \operatorname{Region}(7)$ : There exists a unique $\bar{w} \in B L\left(w_{-}\right)\left(\bar{u}>u_{-}\right)$such that $w_{+} \in$ $R_{2}(\bar{w})$ and the asymptotic state is expected to be

$$
W^{B L}\left(x ; w_{-}, \bar{w}\right)+W_{2}^{R}\left(x / t ; \bar{w}, w_{+}\right)-\bar{w} .
$$

(8) $w_{+} \in \operatorname{Region}(8):$ There exists a unique $\bar{w} \in B L\left(w_{-}\right)\left(\bar{u}>u_{-}\right)$such that $w_{+} \in$ $S_{2}(\bar{w})$ and the asymptotic state is expected to be

$$
W^{B L}\left(x ; w_{-}, \bar{w}\right)+W_{2}^{V S}\left(x-s_{2} t+\alpha ; \bar{w}, w_{+}\right)-\bar{w} .
$$

(9) $w_{+} \in R_{2}\left(w_{*}\right)$ : The asymptotic state is expected to be

$$
W^{B L}\left(x ; w_{-}, w_{*}\right)+W_{2}^{R}\left(x / t ; w_{*}, w_{+}\right)-w_{*} .
$$

(10) $w_{+} \in S_{2}\left(w_{*}\right)$ : The asymptotic state is expected to be

$$
W^{B L}\left(x ; w_{-}, w_{*}\right)+W_{2}^{S}\left(x-s_{2} t+\alpha ; w_{*}, w_{+}\right)-w_{*} .
$$

(11) $w_{+} \in R_{1}\left(w_{*}\right):$ The asymptotic state is expected to be

$$
W^{B L}\left(x ; w_{-}, w_{*}\right)+W_{1}^{R}\left(x / t ; w_{*}, w_{+}\right)-w_{*} .
$$

(12) $w_{+} \in \operatorname{Region}(12)$, i.e., $R R\left(w_{*}\right)$ : There exists a unique $\bar{w} \in R_{1}\left(w_{*}\right)$ such that $w_{+} \in R_{2}(\bar{w})$ and the asymptotic state is expected to be

$$
W^{B L}\left(x ; w_{-}, w_{*}\right)+\left(W_{1}^{R}\left(x / t ; w_{*}, \bar{w}\right)-w_{*}\right)+\left(W_{2}^{R}\left(x / t ; \bar{w}, w_{+}\right)-\bar{w}\right) .
$$

(13) $w_{+} \in$ Region(13), i.e., $R S\left(w_{*}\right)$ : There exists a unique $\bar{w} \in R_{1}\left(w_{*}\right)$ such that $w_{+} \in S_{2}(\bar{w})$ and the asymptotic state is expected to be

$$
W^{B L}\left(x ; w_{-}, w_{*}\right)+\left(W_{1}^{R}\left(x / t ; w_{*}, \bar{w}\right)-w_{*}\right)+\left(W_{2}^{V S}\left(x-s_{2} t+\alpha ; \bar{w}, w_{+}\right)-\bar{w}\right) .
$$


Here note that in the cases (9) to (13), the boundary layer $W^{B L}\left(x ; w_{-}, w_{*}\right)$ is degenerate one. In Matsumura \& Nishihara [13], we succeeded in giving rigorous proofs for some cases. First in the case (5), since the $V^{\prime}$ has a right sign, we can prove that the boundary layer solution $W^{B L}\left(x ; w_{-}, w_{+}\right)$is asymptotically stable. Second in the cases (2)(7), combining the arguments in Kawashima \& Nishibata [1] with that in Liu, Matsumura \& Nishihara [4], we can show that if $\left|w_{+}-w_{-}\right|$is suitably small, the combination of two waves $W^{B L}\left(x ; w_{-}, \bar{w}\right)+W_{2}^{R}\left(x / t ; \bar{w}, w_{+}\right)-\bar{w}$ is asymptotically stable. Finally in the case (12), we can prove that if $\left|w_{+}-w_{*}\right|$ is suitably small the combination of three waves is asymptotically stable ( note $\left|w_{-}-w_{*}\right|$ is not necessarily small). We shall state this case more precisely in the last part of this section. All other cases are basically open. Among them, in the cases (3)(8), we should note that the phase shift $\alpha$ in $W^{B L}\left(x ; w_{-}, \bar{w}\right)+W_{2}^{V S}\left(x-s_{2} t+\alpha ; \bar{w}, w_{+}\right)-\bar{w}$ is explicitly determined by the initial perturbation by the entirely similar argument as that at the end of the Section 3, however the whole proof is not completed yet.

- $w_{-} \in \Omega_{\text {super }}^{+} \quad$ (see Figure 6.2):

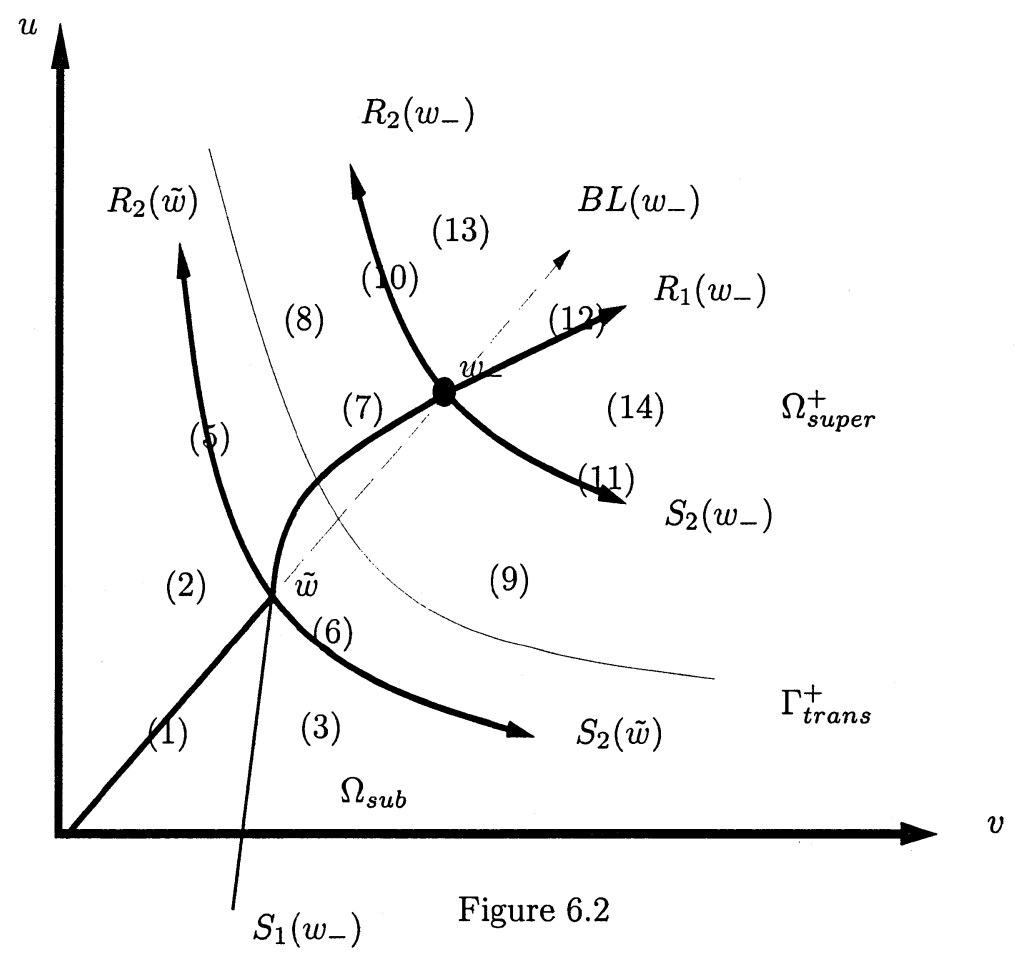

(1) $w_{+} \in B L\left(w_{-}\right), 0<u_{+}<\tilde{u}$ : The asymptotic state is expected to be

$$
W^{B L}\left(x ; w_{-}, w_{+}\right) .
$$

(2) $w_{+} \in \operatorname{Region}(2):$ There exists a unique $\bar{w} \in B L\left(w_{-}\right)$such that $w_{+} \in R_{2}(\bar{w})$ and 
the asymptotic state is expected to be

$$
W^{B L}\left(x ; w_{-}, \bar{w}\right)+W_{2}^{R}\left(x / t ; \bar{w}, w_{+}\right)-\bar{w} .
$$

(3) $w_{+} \in \operatorname{Region}(3)$ : There exists a unique $\bar{w} \in B L\left(w_{-}\right)$such that $w_{+} \in S_{2}(\bar{w})$ and the asymptotic state is expected to be

$$
W^{B L}\left(x ; w_{-}, \bar{w}\right)+W_{2}^{V S}\left(x-s_{2} t+\alpha ; \bar{w}, w_{+}\right)-\bar{w} .
$$

(4) $w_{+}=\tilde{w}:$ The asymptotic state is expected to be

$$
W_{1}^{V S}\left(x+\alpha(t) ; w_{-}, \tilde{w}\right) \text { for some } \alpha(t) \nearrow+\infty .
$$

(5) $w_{+} \in R_{2}(\tilde{w}):$ The asymptotic state is expected to be

$$
W_{1}^{V S}\left(x+\alpha(t) ; w_{-}, \tilde{w}\right)+W_{2}^{R}\left(x / t ; \tilde{w}, w_{+}\right)-\tilde{w}
$$

for some $\alpha(t) \nearrow+\infty$.

(6) $w_{+} \in S_{2}(\tilde{w}):$ The asymptotic state is expected to be

$$
W_{1}^{V S}\left(x+\alpha(t) ; w_{-}, \tilde{w}\right)+W_{2}^{V S}\left(x-s_{2} t+\alpha_{2} ; \tilde{w}, w_{+}\right)-\tilde{w}
$$

for some $\alpha(t) \nearrow+\infty$ and $\alpha_{2} \in \mathbf{R}$.

(7) $w_{+} \in S_{1}\left(w_{-}\right)$: The asymptotic state is expected to be

$$
W_{1}^{V S}\left(x-s_{1} t+\alpha ; w_{-}, w_{+}\right)
$$

(8) $w_{+} \in$ Region(8): There exists a unique $\bar{w} \in S_{1}\left(w_{-}\right)\left(\tilde{u}<\bar{u}<u_{-}\right)$such that $w_{+} \in R_{2}(\bar{w})$ and the asymptotic state is expected to be

$$
W_{1}^{V S}\left(x-s_{1} t+\alpha ; w_{-}, \bar{w}\right)+W_{2}^{R}\left(x / t ; \bar{w}, w_{+}\right)-\bar{w} .
$$

(9) $w_{+} \in \operatorname{Region}(9)$ : There exists a unique $\bar{w} \in S_{1}\left(w_{-}\right)\left(\tilde{u}<\bar{u}<u_{-}\right)$such that $w_{+} \in S_{2}(\bar{w})$ and the asymptotic state is expected to be

$$
W_{1}^{V S}\left(x-s_{1} t+\alpha_{1} ; w_{-}, \bar{w}\right)+W_{2}^{V S}\left(x-s_{2} t+\alpha_{2} ; \bar{w}, w_{+}\right)-\bar{w} .
$$

(10) $w_{+} \in R_{2}\left(w_{-}\right):$The asymptotic state is expected to be

$$
W_{2}^{R}\left(x / t ; w_{-}, w_{+}\right)
$$

(11) $w_{+} \in S_{2}\left(w_{-}\right)$: The asymptotic state is expected to be

$$
W_{2}^{V S}\left(x-s_{2} t+\alpha ; w_{-}, w_{+}\right) .
$$

(12) $w_{+} \in R_{1}\left(w_{-}\right)$: The asymptotic state is expected to be

$$
W_{1}^{R}\left(x / t ; w_{-}, w_{+}\right)
$$

(13) $w_{+} \in$ Region(13), i.e., $R R\left(w_{-}\right)$: There exists a unique $\bar{w} \in R_{1}\left(w_{-}\right)$such that $w_{+} \in R_{2}(\bar{w})$ and the asymptotic state is expected to be

$$
W_{1}^{R}\left(x / t ; w_{-}, \bar{w}\right)+W_{2}^{R}\left(x / t ; \bar{w}, w_{+}\right)-\bar{w} .
$$


(14) $w_{+} \in$ Region(14), i.e., $R S\left(w_{-}\right)$: There exists a unique $\bar{w} \in R_{1}\left(w_{-}\right)$such that $w_{+} \in S_{2}(\bar{w})$ and the asymptotic state is expected to be

$$
W_{1}^{R}\left(x / t ; w_{-}, \bar{w}\right)+W_{2}^{V S}\left(x-s_{2} t+\alpha ; \bar{w}, w_{+}\right)-\bar{w}
$$

Basically the above 14 cases are open. In the cases (7) to (14), we can easily imagine the behaviors of solutions are similar to that for the Cauchy problem since the situation is totally supersonic. However, because of presence of the boundary, the mathematical proofs are not completed yet even in the cases $(10)(12)(13)$ which are completely solved for the Cauchy problem. The cases (4)(5)(6) should be subtle and might be even more difficult than the case (5.7) of the Outflow problem. In the cases $(1)(2)(3)$, it is interesting to see that although supersonic is the state around the boundary, the state at far field is subsonic enough to create an incoming wave to the boundary which eventually forms a boundary layer.

In the remaining part of this section, we state more precisely about the sub-case (12) in the primary case $w_{-} \in \Omega_{s u b}$ where the asymptotic behavior is expected to be a combination of a boundary layer solution and two rarefaction waves. First let us see that by transformation from the Eulerian coordinates $(x, t)$ to the Lagrangian mass coordinates $(\xi, t)$, we can make the original problem easier to handle and become a corresponding IBVP with the moving boundary $\left(x=s_{0} t, s_{0}=-u_{-} / v_{-}<0\right)$. In fact, if we keep in mind that the mass flows in through the boundary at a rate of $\rho_{-} u_{-}$, we may define the transformation $x=x(\xi, t)$ for $\xi \geq 0$ by

$$
\left\{\begin{array}{l}
\frac{\partial x(\xi, t)}{\partial t}=u(t, x(\xi, t)), \quad t>0, \xi>0 \\
x(\xi, t)=x_{0}(\xi)
\end{array}\right.
$$

where $x_{0}(\xi)$ is given by the relation

$$
\xi=\int_{0}^{x_{0}(\xi)} \rho(y, 0) d y
$$

and for $\xi<0$ by

$$
\left\{\begin{array}{l}
\frac{\partial x(\xi, t)}{\partial t}=u(t, x(\xi, t)), \quad t>t_{0}(\xi), \xi<0 \\
x\left(\xi, t_{0}(\xi)\right)=0
\end{array}\right.
$$

where $t_{0}(\xi)$ is given by the relation

$$
-\xi=\int_{0}^{t_{0}(\xi)}(\rho u)(0, \tau) d \tau=\left(\rho_{-} u_{-}\right) \cdot t_{0}(\xi) .
$$

By elementary calculations, we deduce from $(6.1) \sim(6.4)$ that

$$
\xi=\int_{x(\xi, 0)}^{x(\xi, t)} \rho(y, t) d y, \quad \xi \geq s_{0} t
$$

which implies

$$
\frac{\partial x(\xi, t)}{\partial \xi}=v(t, x(\xi, t)), \quad \frac{\partial x(\xi, t)}{\partial t}=u(t, x(\xi, t)), \quad \xi \geq s_{0} t
$$


Due to the relations (6.5), we can rewrite the original problem $(1.1) \sim(1.4)_{3}$ in the following form where we use again $x$ instead of $\xi$ :

$$
\left\{\begin{array}{l}
v_{t}-u_{x}=0 \\
u_{t}+p(v)_{x}=\mu\left(u_{x} / v\right)_{x}, \quad x>s_{0} t, t>0 \\
p(v)=a v^{-\gamma}
\end{array}\right.
$$

with the initial data

$$
(v, u)(0, x)=\left(v_{0}, u_{0}\right)(x), \quad x>0, \quad \inf _{x>0} v_{0}>0,
$$

the boundary condition at far field $x=+\infty$

$$
\lim _{x \rightarrow \infty}(v, u)(t, x)=\left(v_{+}, u_{+}\right), \quad t>0
$$

and also the condition on the moving boundary $x=s_{0} t$

$$
(v, u)\left(t, s_{0} t\right)=\left(v_{-}, u_{-}\right), \quad t>0 .
$$

This time the hyperbolic part of (6.6) is written for smooth $v$ and $u$ in the form

$$
\left(\begin{array}{c}
v \\
u
\end{array}\right)_{t}+A^{L}(v)\left(\begin{array}{c}
v \\
u
\end{array}\right)_{x}=0
$$

where

$$
A^{L}(v)=\left(\begin{array}{cc}
0 & -1 \\
p^{\prime}(v) & 0
\end{array}\right)
$$

It is easy to see that the eigenvalues $\left\{\lambda_{i}^{L}(v)\right\}_{i=1}^{2}$ of $A^{L}(v)$ and corresponding right eigenvectors $\left\{r_{i}\right\}_{i=1}^{2}$ are just the same as in (2.4),

$$
\lambda_{1}^{L}(v)=-\lambda_{2}^{L}(v)=-\left|p^{\prime}(v)\right|^{1 / 2}, \quad r_{i}(v)=\left(\begin{array}{c}
1 \\
-\lambda_{i}^{L}(v)
\end{array}\right)
$$

which implies the rarefaction curves $R_{i}\left(w_{-}\right)$(or $\tilde{R}_{i}\left(w_{+}\right)$) are naturally the same as that for the Eulerian case. As for the Rankine-Hugoniot condition, we have

$$
\left\{\begin{array}{l}
-s\left(v_{+}-v_{-}\right)-\left(u_{+}-u_{-}\right)=0 \\
-s\left(u_{+}-u_{-}\right)+\left(p\left(v_{+}\right)-p\left(v_{-}\right)\right)=0
\end{array}\right.
$$

which implies that

$$
\left\{\begin{array}{l}
u_{+}=u_{-}-\left(v_{+}-v_{-}\right) s_{i}^{L}\left(v_{-}, v_{+}\right), \\
s=s_{i}^{L}\left(v_{-}, v_{+}\right), \quad i=1,2
\end{array}\right.
$$

where $s_{i}^{L}\left(v_{-}, v_{+}\right)$is as in (2.7). Thus the shock curves $S_{i}\left(w_{-}\right)$(or $\left.\tilde{S}_{i}\left(w_{+}\right)\right)$and the line $B L\left(w_{-}\right)$are also naturally the same as in the Eulerian case, excepting the shock speeds $s_{i}\left(w_{-}, v_{+}\right)$are replaced by $s_{i}^{L}\left(v_{-}, v_{+}\right)$. Let us use the same notations $W_{i}^{R}, W_{i}^{S}, W_{i}^{V S}$, 
and $W^{B L}$ to represent rarefaction wave, shock wave, viscous shock wave, and boundary layer solution respectively. Among them, we especially should note that boundary layer solution is no longer stationary, but defined by a travelling wave solution with the same propagation speed $\left(s_{0}=-u_{-} / v_{-}\right)$as the moving boundary, that is, $W^{B L}\left(x-s_{0} t ; w_{-}, w_{+}\right)$is defined by the solution $W(\xi)\left(\xi=x-s_{0} t\right)$ of

$$
\left\{\begin{array}{l}
-s_{0} V^{\prime}-U^{\prime}=0 \\
-s_{0} U^{\prime}+(p(V))^{\prime}=\mu\left(U^{\prime} / V\right)^{\prime}, \quad \xi>0 \\
W(0)=w_{-}, \quad W(+\infty)=w_{+}
\end{array}\right.
$$

which is equivalent to, for $w_{+} \in B L\left(w_{-}\right)$,

$$
\left\{\begin{array}{l}
U=s_{0} V \\
s_{0} \mu V^{\prime} / V=-s_{0}^{2}\left(V-v_{+}\right)+p\left(v_{+}\right)-p(V), \quad \xi>0 \\
V(0)=v_{-}, \quad V(+\infty)=v_{+} .
\end{array}\right.
$$

Since the right hand side of the second equation of (6.15) is exactly the same as that of (4.3), the Proposition 4.1 and 4.2 hold as they are also for (6.15).

Now we fix any $w_{*}$ on $\Gamma_{\text {trans }}^{+}$and suppose $w_{-} \in B L\left(w_{*}\right)\left(u_{-}<u_{*}\right)$ and $w_{+} \epsilon$ $R R\left(w_{*}\right)$. We also assume that the initial data in (6.7) satisfy

$$
w_{0}-w_{+} \in H^{1}\left(\mathbf{R}_{+}\right)
$$

and the compatibility condition

$$
w_{0}(0)=w_{-} \text {. }
$$

Then, as stated above, the solution of the Inflow Problem (6.6) (6.9) is expected to tend toward a combination of three elementary waves

$$
\begin{gathered}
W^{a s y m p}(t, x):=W^{B L}\left(x-s_{0} t, w_{-}, w_{*}\right)+W_{1}^{R}\left(x / t ; w_{*}, \bar{w}\right)-w_{*}+ \\
+W_{2}^{R}\left(x / t ; \bar{w}, w_{+}\right)-\bar{w} .
\end{gathered}
$$

Roughly speaking, since the asymptotic state $W^{a s y m p}$ has a right sign (that is, $V_{t}^{\text {asymp }}=U_{x}^{a s y m p}>0$ ) to adapt the $L^{2}$ energy method, we can prove the following theorem by combining the arguments in Matsumura \& Nishihara [12], how to handle the rarefaction waves for the viscous p-system, together with that in Liu, Matsumura \& Nishihara [4], how to dispose the interactions between rarefaction waves and boundary layer solutions.

Theorem 6.1. (Matsumura \& Nishihara [13]) Suppose $w_{*} \in \Gamma_{\text {trans }}^{+}, w_{-} \in$ $B L\left(w_{*}\right)\left(u_{-}<u_{*}\right)$ and $w_{+} \in R R\left(w_{*}\right)$. Assume also (6.16) and (6.17). Then, for any fixed $w_{*}$ and $w_{-}$, there exists a positive constant $\delta_{0}$ such that if $\| w_{0}-\left(W^{B L}-w_{*}\right)-$ $w_{+} \|_{H^{1}}+\left|w_{+}-w_{*}\right|<\delta_{0}$, then the Inflow Problem (6.6) $\sim(6.9)$ has a unique time global solution $w$ satisfying $w-w_{+} \in C\left([0, \infty) ; H^{1}\right)$ and the asymptotic behavior

$$
\lim _{t \rightarrow \infty} \sup _{x \geq s_{0} t}\left|w(t, x)-W^{a s y m p}(t, x)\right|=0 .
$$


Finally let us comment on some future's problems. We first should extend the above arguments to the full system including the conservation of energy. We next should extend the arguments to cases, even a $2 \times 2$ viscous p-system model, with a free boundary on which inflow or outflow occurs as a result of phase transitions, chemical reactions, etc. Eventually, we hope we could unify the arguments of fluid dynamical aspects and that of Stephan type problems, for example, hopefully could argue on the interactions of free boundary of phase and fluid dynamical waves as shock waves, rarefaction waves and contact discontinuities.

Acknowledgement. The author wishes to thank Prof. S.-H. Yu for his helps to make the Figures.

\section{REFERENCES}

[1] S. Kawashima And S. Nishibata, Stability of stationary waves for the compressible NavierStokes equations in the half space, to appear.

[2] T.-P. LIU, Nonlinear stability of shock waves for viscous conservation laws, Mem. AMS, 56 (1985).

[3] T.-P. Liu, Shock waves for compressible Navier-Stokes equation are stable, Commun. Pure Appl. Math., 39 (1986), pp. 565-594.

[4] T.-P. LiU, A. MATSUmURA AND K. Nishihara, Behavior of solutions for the Burgers equations with boundary corresponding to rarefaction waves, SIAM J. Math. Anal., 29 (1998), pp. 293-308.

[5] T.-P. LiU AND K. Nishihara, Asymptotic behavior for scalar viscous conservation laws with boundary effect, J. Diff. Eqns., 133 (1997), pp. 296-320.

[6] T.-P. LiU AND S.-H. YU, Propagation of a stationary shock layer in the presence of a boundary, Arch. Rat. Mech. Anal., 139 (1997), pp. 57-82.

[7] A. Matsumura, Large-time behavior of one-dimensional motions of compressible viscous gas, in Recent Developements in Theoretical Fluid Mechanics, Winter School, Paseky, 1992(edited by G.P.Galdi and J.Necas), Longman Sci. Tech., New York, pp. 103-128.

[8] A. Matsumura AND M. MEI, Convergence to travelling fronts of solutions of the p-system with viscosity in the presence of a boundary, Arch. Rat. Mech. Anal., 146 (1999), pp. 1-22.

[9] A. MATSUMURA AND K. NishinARA, On the stability of travelling wave solutions of a onedimensional model system for compressible viscous gas, Japan J. Appl. Math., 2 (1985), pp. $17-25$.

[10] A. MatsumURa AND K. Nishihara, Asymptotics toward the rarefaction waves of the solutions of a one-dimensional model system for compressible viscous gas, Japan J. Appl. Math., 3 (1986), pp. 1-13.

[11] A. MATSUmURA AND K. NishinARA, Global stability of the rarefaction wave of a onedimensional model system for compressible viscous gas, Commn. Math. Phys., 165 (1992), pp. $325-335$.

[12] A. Matsumura and K. Nishinara, -it Global asymptotics toward the rarefaction wave for solutions of viscous $p$-system with boundary effect, to appear in Quart. Appl. Math. (1999).

[13] A. MATSUmURA AND K. Nishihara, Large-time behaviors of solutions to an infow problem in the half space for a one-dimensional system of compressible viscous gas, to appear.

[14] A. SzEPESSY AND Z. XiN, Nonlinear stability of viscous shock waves, Arch. Rat. Mech. Anal., 122 (1993), pp. 53-103.

[15] A. SzePESSY, On the stability of Broadwell shocks, AMS/IP Studies in Advanced Math., 3 (1997), pp. 403-412. 